\title{
EVALUATION OF FATIGUE AND CORROSION TEST OF AI LM13 REINFORCED WITH HEMATITE
}

\author{
Sanju $\mathbf{H}^{\mathbf{1}}$, Meghani Molies Reddy ${ }^{2}$, Batluri Tilak Chandra ${ }^{3}$, Madhu B $\mathbf{P}^{\mathbf{4}}$ \\ ${ }^{1}$ Assistant Professor, Mechanical Engineering, YDIT,Bangalore Karnataka, India \\ ${ }^{2}$ Assistant Professor, Mechanical Engineering, SIET Tumakuru, Karnataka, India \\ ${ }^{3}$ Assistant Professor, Mechanical Engineering, SSIT Tumakuru, Karnataka, India \\ ${ }^{4}$ Assistant Professor, Mechanical Engineering, YDIT ,Bangalore Karnataka, India
}

\begin{abstract}
Aluminum alloys are generally recycled in automotive and aerospace manufacturing due to then improved mechanical properties, better resistance to corrosion and little density, little thermal coefficient extension as equated conservative alloys. The outstanding properties of these constituents and comparatively small manufacture cost make them a same striking aspirant for variation presentation from both technical and systematic perspectives. The objective intricate in designing metallic matrix composite constituents is to syndicate the required characteristics of metal and earthenware. In the current examination the strength of composite AL LM13- hematite calculated by variable the proportion of strengthening fatigue and corrosion test achieved and associated with the strength of heat treated specimens with the same compositions and calculated the fatigue strength of the composite containing microstructure analysis using photosensitive microscopy of the specimen invented with hematite particulates of $150 \mu$ in dissimilar compositions such as $0 \%, 3 \%, 6 \%, 9 \%, 12 \%, 15 \%$ by using stirring cast technique.
\end{abstract}

Keywords: Al LM13, Hematite, Fatigue and Corrosion Test, Stir Casting. $* * *$

\section{INTRODUCTION}

In know a day's composite a major materials role in the developed play of automobiles and aerospace applications. Some of them like rock, wood, stones, etc. As they established in natural process. Characteristic components are the best specialized and appropriate engineering materials. The composition materials are materials prepared from two or more mixture of materials that improved properties of outcomes than those of singular components used unaided for ex; RCC is made up of steel, cement, sand, etc. If the arrangement follows on a microscopic scale the current materials is then called polymers for plastics. Normally the composition materials area arrangement of reinforcement phase fibers, particles lodged solidly in to a matrix of ceramics, polymers, and metals. The matrix seized the reinforcement to methods the produce the shape of whole mechanical properties to increase the reinforcement of the matrix. When produced correctly the original mixture materials give improved strength than each other distinct materials.

Metal matrix composites are normally used in several applications with aerospace and automotive parts, customer and electronic product. It can be arranged by canonical techniques such as injection molding, powder metallurgy, stir casting to make parts of aerospace and automotive applications. Copper, aluminum, titanium, magnesium and nickel based super alloys are presently used in matrix. The aluminum, titanium, magnesium are the three matrices are based alloy for aerospace and automobile applications the reduction in weight and strength concerning this applications are used in electrical contacts, thermal management and copper based matrix composites. For high temperature application the stain less steel and nickel based matrix are used.

Present-day works reveals that in numerous composite applications the hematite established inexpensive performance, the most capable applications the hematite are in metal matrix composites that can molded in to a variability of flat and complex formed components are produced. The possible of nonferrous and ferrous alloy reinforcement composite materials are construct walls with the improvement of more materials such as natural material decreased.

\subsection{Over View of Metal Matrix Composites}

Metal matrix composites similar totally compositions it contains of a minimum two bodily and chemically segment of distinct, it's only appropriate for to deliver the dispersed properties and specific phases are not obtainable. Usually around are a two phases particulate or fibrous segment in a metallic matrix for ex; AL2O3 copper matrix reinforced with fiber for excellent leading magnets. Aluminum matrix composites reinforced with Sic particles used in thermal management, aerospace and automobile applications. 


\section{LITERATURE SURVEY}

C S Ramesh et al., In this author examined Al 6061 aluminum alloy metal matrix composites reinforced with $\mathrm{Ni}$ $\mathrm{P}$ covered Si3N4 strengthened Al 6061 composite were designed stirring cast technique. Silicon nitride components numerous stages in 2 that is 4 wt. $\%$ to $10 \mathrm{wt}$. \%. This casted alloy and established composite existed hot forged temperature of 500 using a $300 \mathrm{~T}$ hydraulic mallet. In this paper investigated microstructure and fatigue strength test. This paper revels that increase the contented of reinforcement in both as cast and hot forged composite does the consequence in significant to development the fatigue strength and associated to the as cast alloy and its composites, and hot forge alloy and its composites. The hot forged alloy and its composite greater fatigue strength [1].

B. Babu et al., In this author present base metal and covered stage for the current studies was Al LM 13 metal matrix composite and reinforced with silicon carbide using stirring cast technique. And the difference of percentage composition $\mathrm{SiCp}-10 \%$ with aluminum. In this paper tested as corrosion test, hardness test and SEM analysis. The result will shows in this paper the composite can be used in sodium chloride atmosphere covering the corrosion rate falls with the mentioned corrosion range 1-200mmpy for generally strong materials [2].

D. Ramesh et al.,In this authors examined to improve the $\mathrm{Al}$ 6061- frit particulate composites over stirring cast technique and to study the corrosion performance. Aluminum 6061 comprising $2 \mathrm{wt} \%$ to $8 \mathrm{wt} \%$ stages of $2 \mathrm{wt} \%$ the corrosion test is conducted $238 \mathrm{C}$ where $3.5 \%$ Nacl solution used as corrode. In this paper revels that the metallic matrix composites corrosion rate stood less than matrix material. Matrix material Al 6061 this paper was showing the investigate on both heat treated and un heat treated [3].

\subsection{Summary of Literature Review}

In existing examination the strong point of composite $\mathrm{Al}$ LM 13-hematite considered by variable the percentage of strengthening and equated with the strength of heat treated specimens with the similar composition and estimated the corrosion test of the composite containing microstructure study by means of photosensitive microscopy of the specimen made-up with hematite particulates of $150 \mu$ in dissimilar arrangements such as $0 \%, 3 \%, 6 \%, 9 \%, 12 \%$ and $15 \%$ by using stirring cast technique.

\subsection{Objectives}

1. To determine fatigue and corrosion test of $\mathrm{Al} \mathrm{LM} 13$ reinforced with hematite for different compositions at $0 \%$, $3 \%, 6 \%, 9 \%, 12 \%, 15 \%$.
2. To design and fabrication of the material using stir casting method

3. The test will be conducted in both heat treated and un heat treated condition specimens.

4. The specimen was heated at normalizing temperature $530^{\circ}$ $\mathrm{c}$ and aging temperature $175^{\circ} \mathrm{c}$ at air quenched.

\subsection{Methodology}

The aluminum LM 13 alloy is a matrix substantial with the chemical composition as given table3.1 was using as the founded alloy. The hematite particulate size $150 \mu$ is used as the reinforcement with the chemical composition as given. The stir casting technique is used to construct the metallic matrix composite. The composites with changed compositions of reinforcements like hematite particulates 0$15 \%$ in odd $\%$ by weight were arranged with the resulting procedure.

1. Melting the aluminum LM 13 alloy in a heater.

2. Arranged and pre heated of the reinforcements.

3. De- gassing and elimination of slag.

4. Mixed the metal and reinforcing material.

5. Bucketing the molten metal matrix and reinforcement mix to the die.

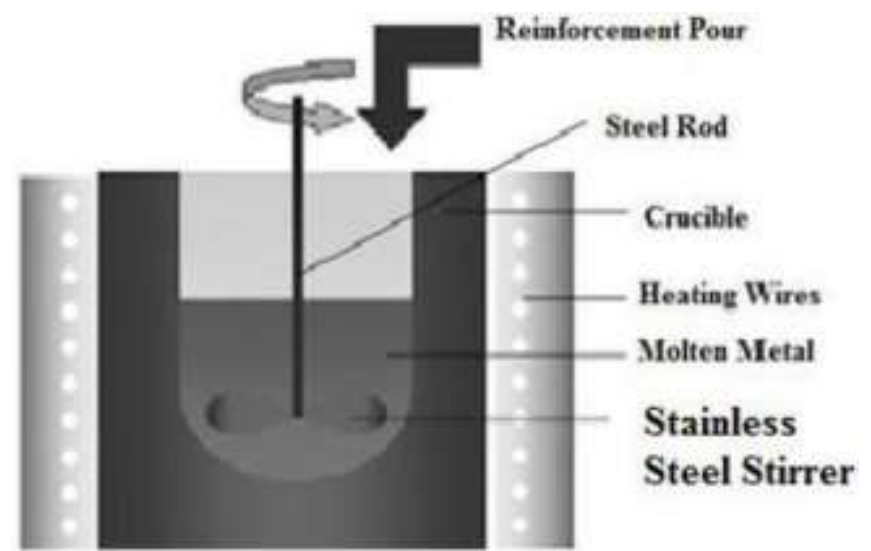

Fig 1.1 Stir Casting Method

For melted the alloy an electric arc furnace is mainly used. The container type heater and Heated electrically at 3 phase resistance of $12.5 \mathrm{~kW}$ capabilities. The heater range is $1200 \mathrm{C}$ with precision $+/-1.5 \mathrm{C}$. The aluminum alloy earlier melted the vessels dead to the cracking. The aluminum LM 13 is cut in to small bits evaluated rendering to the essential and additional to the pre heated vessel. Once the vessel has been changed, switch on the heater $750^{\circ} \mathrm{C}$ temperature as setup. 


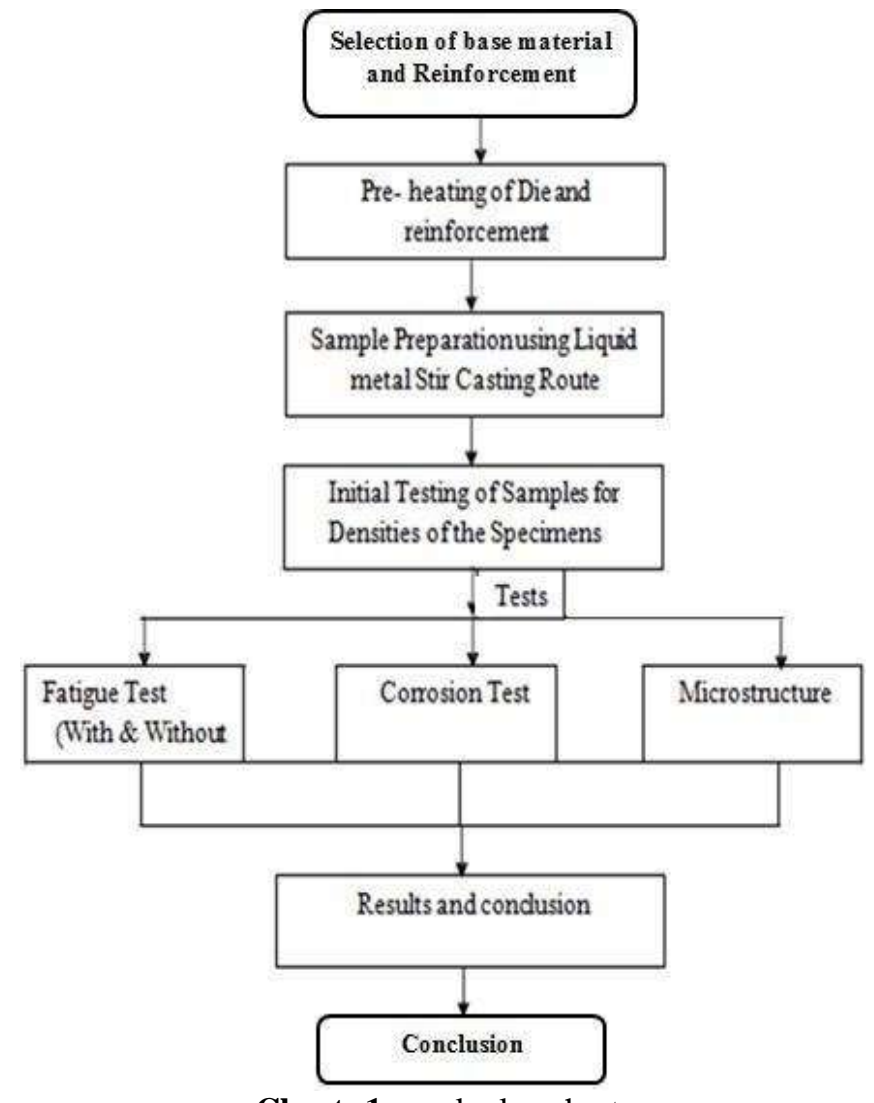

Chart -1: work plan chart

\subsubsection{Fatigue Test}

The as cast and heat treated Al LM13-hematite composites, it is detected that in both as cast and heat treated situations. Fatigue strength of Al LM13-hematite composites is greater than their un-reinforced alloy. Adding of hematite elements improved the fatigue life of the alloy expressively. After the fatigue strength of the composite improved with improve in the contented of hematite elements in both as cast and heat treated situation. The development in the fatigue strength of the developing mixtures are important at lesser stress level than the greater stress level in both as cast and heat treated composites.

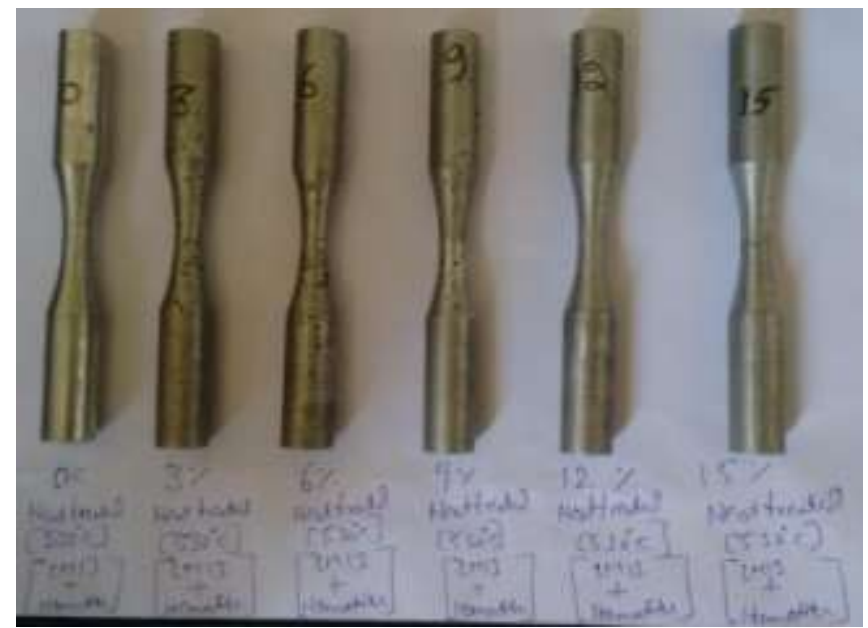

Fig: 1.2 Fatigue test specimens
In both the cases the maximum heaviness proportion of hematite elements exposed significant development in fatigue strength of the composites when equated with the un strengthened alloy. This can be recognized to existence of solid hematite elements. The constant size and delivery of hematite elements over the composites improve the plastic strain essential to reason crack origination in the composite substantial. The fatigue strength growths with volume fraction of the elements since of the important quantity of the weight existence transmitted to the harder particulate strengthening and over all lesser overall strains for a specified fatigue stress.

\subsubsection{Corrosion Test}

The corrosion testing is conducted the cylinder-shaped specimen of dia $20 \mathrm{~mm}$ and height $20 \mathrm{~mm}$ existed machining from castings of the combination and the base alloy. Earlier corrosion testing, the specimen surface existed pounded with 1000 gravel emery paper and then refined by means of $3 \mu \mathrm{m}$ diamond adhesive to achieve a better surface quality. The specimens were then splashed in purified water, following by acetone, and then allowable dry systematically.

The corrosion test existed stationary dipping test lead at room temperature using the conservative weight loss method to an precision of $0.1 \mathrm{mg}$. every sample was first weighed earlier being submerged in $200 \mathrm{ml}$ of Nacl resolution and later occupied out after 24, 48, 72, and 96 hours individually. Nacl solution was designated as the destroying for quicker results subsequently the corrosion readings described in this project qualified formerly. Afterward every corrosion test, the specimen was dipped in Clark resolution for $10 \mathrm{~min}$ and gradually washed with a soft brush to detached followed scale. Afterward ventilation systematically, the specimens were considered another time. The weight loss was restrained and improved in to corrosion rate expressed mille meter per year (mmpy).

\subsubsection{Heat Treated and Aging}

To learning the result of non-natural aging on the composites, the heat treated specimens stood exposed to resolution heat treatment at $530 \mathrm{C}$ for $6 \mathrm{~h}$. quenched in water and then aging at $175 \mathrm{C}$ for $6 \mathrm{~h}$.

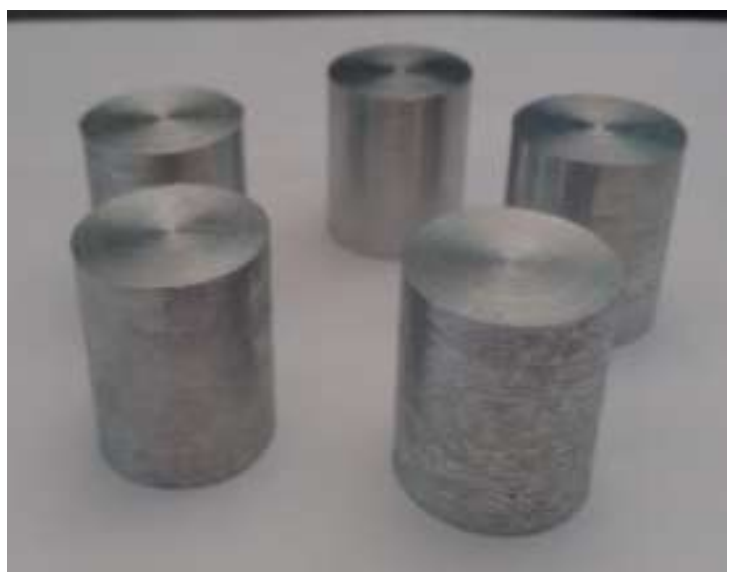

Fig: 1.3 Corrosion test specimens 


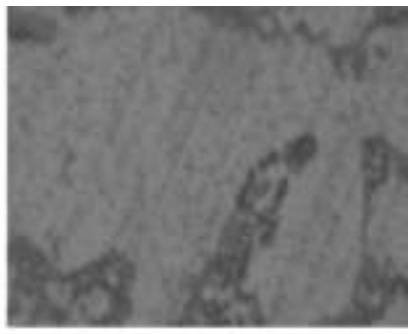

all.M13-Hemable as cast beat traied

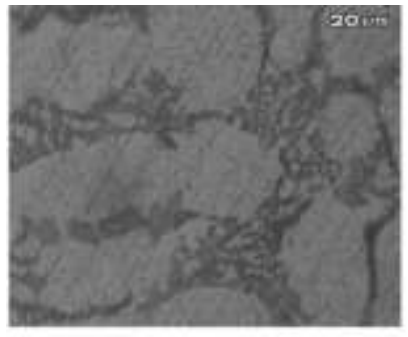

Nin.M3 as caste
Fig 1.4 Shows microstructure of as cast un heat treated and heat treated Al LM13-Hematite

\section{RESULTS AND DISCUSSION}

The Graph 1.1 shows stress v/s number of cycles for $\mathrm{Al}$ LM13-Heamite particulate composites for un heat treated specimen it shows that decreasing number cycles with increasing stresses for the different compositions.

\subsection{Fatigue Test}

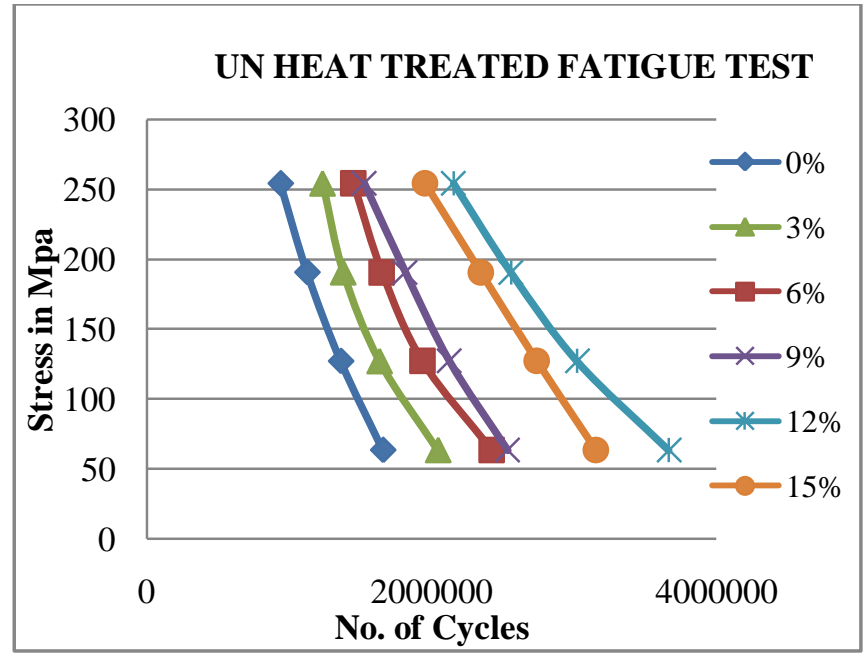

Graph 1.1 Stress v s Number of cycles for Un heat treated Fatigue Specimens

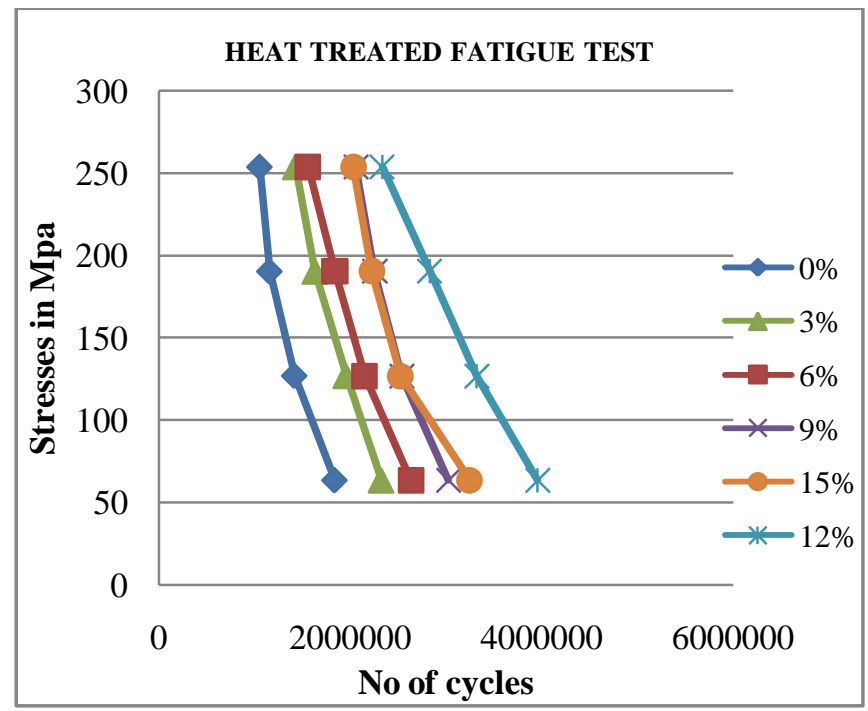

Graph 1.2 Stress v/s Number of cycles for heat treated Fatigue Test Specimens
From both graphs concluded that the fatigue strength increases in heat treated specimen compare to un heat treated specimen.

When the Composition percentage increases the fatigue strength also increases.

\subsection{Corrosion Test}

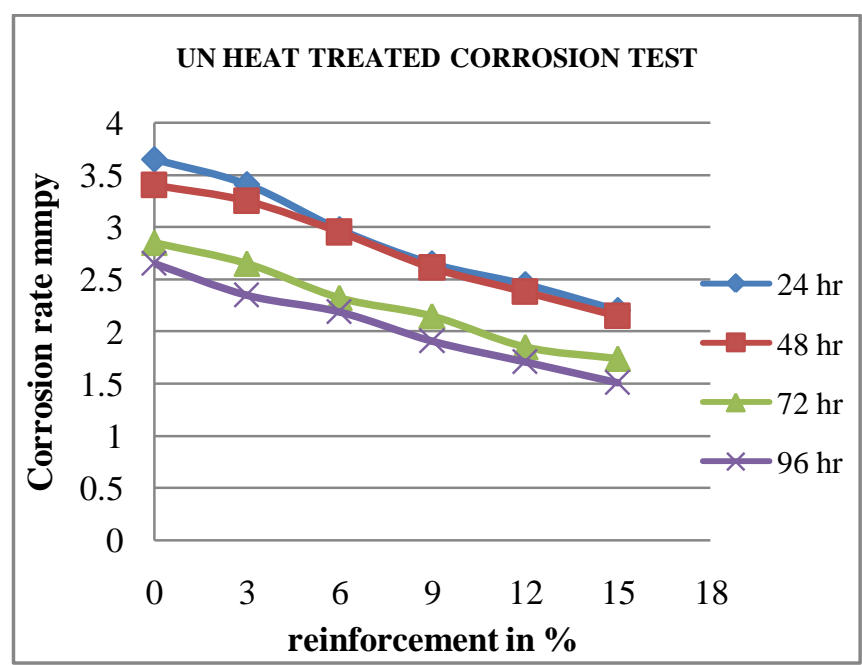

Graph 1.3 Corrosion rate v/s reinforcement in \%

Graph 1.3 shows of Corrosion rate v/s reinforcement in \% in Un heat treated specimens it shows when corrosion from the graph is prove that when the $\%$ of reinforcement increases the corrosion rate was decreases.

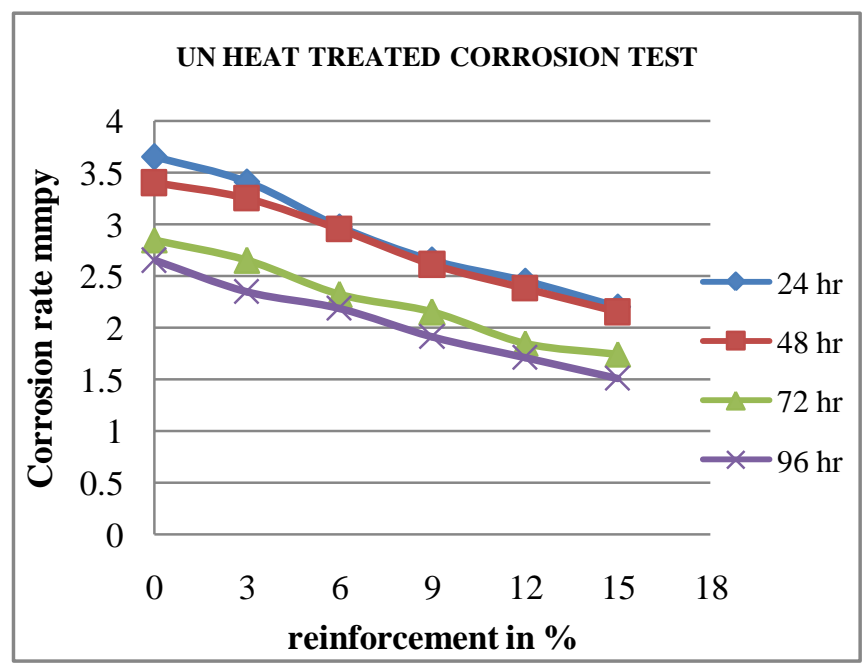

Graph 1.4 Corrosion rate v/s reinforcement in \% for heat treated

Graph 1.4 shows of Corrosion rate v/s reinforcement in \% in Un heat treated specimens it shows when corrosion from the graph is prove that when the \% of reinforcement increases the corrosion rate was decreases.

When compare to both the cases heat treated corrosion test specimens the corrosion rate decreases compare to the un heat treated specimen 


\section{CONCLUSIONS}

The current effort on creation and description of aluminum LM13 -hematite composites Directed to followed conclusions, they are

1. The stirring cast technique to organize the composites might create constant supply of the strengthened hematite elements of weight percentages from 0 to $15 \%$ in odd stages.

2. MMC of aluminum strengthened hematite particulates are established to have the increase fatigue strength as improved in weight percentage and also aluminum LM13 alloy only. Hence the MMC designed in greater to aluminum LM13.

3. Heat treated fatigue specimen show higher fatigue strength than casted specimen.

4. The fatigue strength increases up to the $12 \%$ of reinforcement and fatigue strength decreases $15 \%$ of reinforcement.

5. Corrosion rate value reductions as reinforcement wt. $\%$ growths.

6. Heat treated corrosion test specimen show decreases the corrosion rate than casted specimen.

7. It is detected that density of the composite reductions on adding of hematite.

8. Microstructure photomicrographs show the constant supply of hematite in aluminum matrix.

\section{ACKNOWLEDGEMENT}

The personal satisfaction and the value of any project work remains incomplete without a vote of thanks to all the people involved in the project, directly or indirectly. Here by I would like to express my gratitude to the people who have been a source of constant support, encouragement and guidance throughout my project.

First of all I wish to thank Dr. NALANI N, Principal, SIET, and Tumkur, for having inspired and encouraging us throughout my PG course.

I am grateful to Dr. Sathisha N, HOD, Dept. of Mechanical Engineering, SIET, Tumkur, for sharing his knowledge and experience with us and his valuable advice and supervision at every stage of the project.

I convey my thanks to Mr. H Rangaswamy, Asst. Professor, Dept. of Mechanical Engineering, and PG coordinator and also to Mr.Meghani Molies Reddy, Asst. Professor, Dept. of Mechanical Engineering, my project guide, Dept of ME SIET, Tumkur.

Finally, I would like to express my sincere thanks to our beloved parents, family members, and friends and all Teaching and Non-teaching Staff members of Department of Mechanical Engineering, SIET, Tumkur for their valuable guidance and support.

\section{REFERENCES}

[1]. C. S. Ramesh. R Keshavmurthy and J Mad Ni-P Coated Si3N4 Reinforced Al 6061 Composites.

[2]. B Babu.S K Karthikeyan and V adithya D Ramesh. R P Swami K Chandrasekhar analysis of corrosion and hardness using "Frit particulate composite in sodium chloride solution" Sep 2012 Sri Siddhartha institute of Technology Tumakuru.

[3]. Manoj Singla, D.Deepak Dwivedi, Lakhvir Singh, Vikas Chawla. Development of Aluminum Based Silicon Carbide Particulate Metal Matrix Composite, Journal of Minerals\& Materials Characterization \& Engineering, Vol. 8, No.6, pp. 455-467, 2009[2009].

[4]. N.L.Han,Z.G.Wang,G.D.Zhang, $1997^{\mathrm{c}}$ Effectedtemperat ure tensile properties and low cycle fatigue behavior of particulate $\mathrm{SiC} / \mathrm{Al}$ composite" Composite Science And Technology 